\title{
2D-DOA and Mutual Coupling Estimation in Vehicle Communication System via Conformal Array
}

\author{
Yan Zou, ${ }^{1,2}$ Hong Xie, ${ }^{1}$ Liangtian Wan, ${ }^{3}$ Guangjie Han, ${ }^{3}$ and Wei Li ${ }^{3}$ \\ ${ }^{1}$ College of Information \& Communication Engineering, Harbin Engineering University, Harbin 150001, China \\ ${ }^{2}$ No. 91404 Army, Qinhuangdao 066000, China \\ ${ }^{3}$ Department of Information and Communication Systems, Hohai University, Changzhou 213022, China
}

Correspondence should be addressed to Guangjie Han; hanguangjie@gmail.com

Received 29 September 2015; Accepted 1 December 2015

Academic Editor: Jong-Hyouk Lee

Copyright (C) 2015 Yan Zou et al. This is an open access article distributed under the Creative Commons Attribution License, which permits unrestricted use, distribution, and reproduction in any medium, provided the original work is properly cited.

\begin{abstract}
Many direction-of-arrival (DOA) estimation algorithms have been proposed recently. However, the effect of mutual coupling among antenna elements has not been taken into consideration. In this paper, a novel DOA and mutual coupling coefficient estimation algorithm is proposed in intelligent transportation systems (ITS) via conformal array. By constructing the spectial mutual coupling matrix (MCM), the effect of mutual coupling can be eliminated via instrumental element method. Then the DOA of incident signals can be estimated based on parallel factor (PARAFAC) theory. The PARAFAC model is constructed in cumulant domain using covariance matrices. The mutual coupling coefficients are estimated based on the former DOA estimation and the matrix transformation between MCM and the steering vector. Finally, due to the drawback of the parameter pairing method in Wan et al., 2014, a novel method is given to improve the performance of parameter pairing. The computer simulation verifies the effectiveness of the proposed algorithm.
\end{abstract}

\section{Introduction}

Intelligent transportation systems (ITS) have emerged as an effective way of improving the performance of transportation systems and enhancing travel security. The term connected vehicles refers to applications, services, and technologies that connect a vehicle to its surroundings. With the rapid development of 5G, the peak data rate will likely be in the range of tens of Gbps, which is suitable for real-time communication between vehicles and BS (base station). In order to minimize the cost of the vehicles and reduce the friction resistance between the surface of the vehicles and atmosphere, the conformal array antennas installed in the vehicles should be a good choice.

Direction-of-arrival (DOA) estimation plays an important role in array signal processing, which has been widely used in radar, sonar, and smart antenna [1-3]. Multiple signal classification- (MUSIC-) based algorithm [4] and estimation of signal parameters via rotational invariance techniques(ESPRIT-) based algorithm [5] are two types of DOA estimation algorithms which can achieve superresolution. However, the effect of mutual coupling will reduce the performance of DOA estimation severely.

In order to solve this problem, many algorithms have been proposed to deal with it. An effective compensation method for the effect of mutual coupling in uniform circular arrays (UCAs) employed for two-dimensional (2D) DOA estimations was proposed [6]. A DOA estimation algorithm for mixed signals with unknown mutual coupling was proposed in [7]. The noncoherent (uncorrelated or partially correlated) signals were firstly estimated with unknown mutual coupling; then the mutual coupling coefficients can be obtained by these estimation. Finally, the noncoherent signals were eliminated and the effect of mutual coupling was compensated. The bias in uniform linear array (ULA) and general linear array caused by effect of mutual coupling was studied in [8]. The DOA estimation for noncoherent and coherent signals has been proposed in [9], which is used for patient's localization.

Parallel factor (PARAFAC) analysis attracted the attention of researchers when it was originally introduced in array signal processing in 2000 [10, 11]. It has been widely used for low-rank decomposition of three-way and higher way array. 


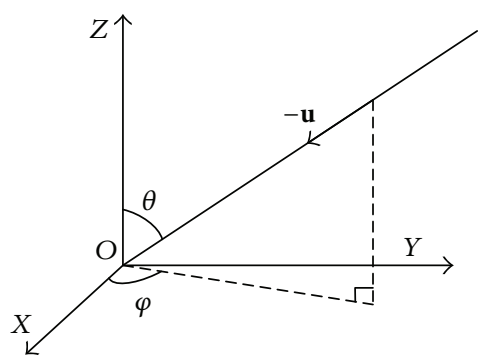

(a)

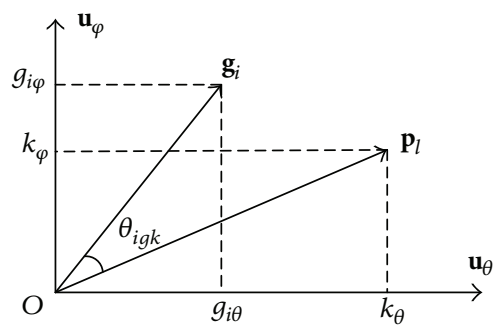

(b)

Figure 1: (a) The incident signal u. (b) The ith element's response.

Based on PARAFAC and cumulant, a new array structure was designed for 2D-DOA estimation [12]. The DOA and polarization estimation for a single electromagnetic vector-sensor were acquired by using PARAFAC [13], and the proposed algorithm performs better than the ESPRIT algorithm.

Conformal array is the array integrated on surface of object $[14,15]$. Some DOA estimation algorithms have been proposed for conformal array. The propagator method and three rotational invariance relationships have been used for fast DOA estimation for cylindrical conformal array [16]. A ultrawideband DOA estimation algorithm has been proposed for DOA estimation based on spatial baseline method and multiple subarray technique [17]. The manifold separation technique has been used for DOA estimation of wireless sensor network with arbitrary array configuration $[18,19]$. A real-time DOA estimation algorithm has been proposed in [20]. The DOA estimation is accomplished via ESPRIT and the elimination of the effect of mutual coupling [21]. Based on spatial baseline technique, the DOA can be estimated with low computational complexity. However, only one source can be estimated [22]. In [23], the authors have extended the PARAFAC theory for frequency and DOA estimation. By using space-time matrix technique, the frequency and DOA estimation have been completed as well. However, the positions of four guiding elements have to be known as a prior [24].

In this paper, a novel DOA and mutual coupling coefficient estimation algorithm for conformal array is proposed in vehicle communication system. In realistic application, the effect of mutual coupling should not be ignored. Based on the selection matrix, the spectial mutual coupling matrix (MCM) is constructed. The effect of mutual coupling can be eliminated by using the spectial MCM. Then two rotational invariant matrices are constructed in order to estimate the DOA of incident signals based on conformal array. Based on PARAFAC theory, the model is constructed in cumulant domain using covariance matrices. The mutual coupling coefficient is estimated finally via matrix transformation.

\section{The Snapshot Data Model}

The algorithm performance would deteriorate significantly because of the mutual coupling among different elements. In order to estimate DOA estimation accurately, the effect of mutual coupling should be considered in the snapshot data model of the conformal array. Due to the varying curvature of carriers, each element possesses different patterns [18]. The incident signals are shown in Figure 1(a) with elevation $\theta$ and azimuth $\varphi$. We can give the form of steering vector as follows:

$$
\begin{aligned}
& \mathbf{a}(\theta, \varphi)=\left[h_{1} e^{-j 2 \pi\left(\left(\mathbf{p}_{1} \cdot \mathbf{u}\right) / \lambda\right)}, h_{2} e^{-j 2 \pi\left(\left(\mathbf{p}_{2} \cdot \mathbf{u}\right) / \lambda\right)}, \ldots,\right. \\
& \left.h_{2 M} e^{-j 2 \pi\left(\left(\mathbf{p}_{2 M} \cdot \mathbf{u}\right) / \lambda\right)}\right]^{T}, \\
& h_{m}=\left(g_{m \theta}^{2}+g_{m \varphi}^{2}\right)^{1 / 2}\left(k_{\theta}^{2}+k_{\varphi}^{2}\right)^{1 / 2} \cos \left(\theta_{m g k}\right)=\left|g_{m}\right| \\
& \cdot\left|p_{l}\right| \cos \left(\theta_{m g k}\right)=\mathbf{g}_{m} \cdot \mathbf{p}_{l}=g_{m \theta} k_{\theta}+g_{m \varphi} k_{\varphi} .
\end{aligned}
$$

The notation of alphabet mentioned above can be found in [25]. Taking (2) into (1), we have

$$
\mathbf{a}(\theta, \varphi)=\mathbf{a}_{\theta}(\theta, \varphi)+\mathbf{a}_{\varphi}(\theta, \varphi)
$$

where

$$
\begin{aligned}
& \mathbf{a}_{\theta}(\theta, \varphi)=\left[g_{1 \theta} k_{\theta} e^{-j 2 \pi\left(\left(\mathbf{p}_{1} \cdot \mathbf{u}\right) / \lambda\right)}, g_{2 \theta} k_{\theta} e^{-j 2 \pi\left(\left(\mathbf{p}_{2} \cdot \mathbf{u}\right) / \lambda\right)}, \ldots,\right. \\
& \left.g_{2 M \theta} k_{\theta} e^{-j 2 \pi\left(\left(\mathbf{p}_{2 M} \cdot \mathbf{u}\right) / \lambda\right)}\right]^{T}, \\
& \mathbf{a}_{\varphi}(\theta, \varphi)=\left[g_{1 \varphi} k_{\varphi} e^{-j 2 \pi\left(\left(\mathbf{p}_{1} \cdot \mathbf{u}\right) / \lambda\right)}, g_{2 \varphi} k_{\varphi} e^{-j 2 \pi\left(\left(\mathbf{p}_{2} \cdot \mathbf{u}\right) / \lambda\right)}, \ldots,\right. \\
& \left.g_{2 M \varphi} k_{\varphi} e^{-j 2 \pi\left(\left(\mathbf{p}_{2 M} \cdot \mathbf{u}\right) / \lambda\right)}\right]^{T} .
\end{aligned}
$$

When $P$ incident signals arrive in the array, the data matrix of array output can be expressed as

$$
\begin{aligned}
\mathbf{X}(n) & =\mathbf{G} \odot \mathbf{C A S}(n)+\mathbf{N}(n) \\
& =\mathbf{C}\left(\mathbf{G}_{\theta} \odot \mathbf{A}_{\theta} \mathbf{K}_{\theta}+\mathbf{G}_{\varphi} \odot \mathbf{A}_{\varphi} \mathbf{K}_{\varphi}\right) \mathbf{S}(n)+\mathbf{N}(n) \\
& =\mathbf{C B S}(n)+\mathbf{N}(n), \\
\mathbf{G}_{\theta} & =\left[\mathbf{g}_{\theta}\left(\theta_{1}, \varphi_{1}\right), \mathbf{g}_{\theta}\left(\theta_{2}, \varphi_{2}\right), \ldots, \mathbf{g}_{\theta}\left(\theta_{P}, \varphi_{P}\right)\right], \\
\mathbf{G}_{\varphi} & =\left[\mathbf{g}_{\varphi}\left(\theta_{1}, \varphi_{1}\right), \mathbf{g}_{\varphi}\left(\theta_{2}, \varphi_{2}\right), \ldots, \mathbf{g}_{\varphi}\left(\theta_{P}, \varphi_{P}\right)\right],
\end{aligned}
$$




$$
\begin{aligned}
\mathbf{A}_{\theta} & =\left[\mathbf{a}_{\theta}\left(\theta_{1}, \varphi_{1}\right), \mathbf{a}_{\theta}\left(\theta_{2}, \varphi_{2}\right), \ldots, \mathbf{a}_{\theta}\left(\theta_{P}, \varphi_{P}\right)\right], \\
\mathbf{A}_{\varphi} & =\left[\mathbf{a}_{\varphi}\left(\theta_{1}, \varphi_{1}\right), \mathbf{a}_{\varphi}\left(\theta_{2}, \varphi_{2}\right), \ldots, \mathbf{a}_{\varphi}\left(\theta_{P}, \varphi_{P}\right)\right], \\
\mathbf{K}_{\theta} & =\operatorname{diag}\left(k_{1 \theta}, k_{2 \theta}, \ldots, k_{P \theta}\right), \\
\mathbf{K}_{\varphi} & =\operatorname{diag}\left(k_{1 \varphi}, k_{2 \varphi}, \ldots, k_{P \varphi}\right), \\
\mathbf{S}(n) & =\left[s_{1}(n), s_{2}(n), \ldots, s_{P}(n)\right]^{T}, \\
\mathbf{W}(n) & =\left[w_{1}(n), w_{2}(n), \ldots, w_{2 M}(n)\right]^{T},
\end{aligned}
$$

where $\mathbf{G}$ denotes the pattern matrix and $\mathbf{A}$ denotes the manifold matrix. $\odot$ stands for the Kronecker product. $k_{1 \theta}, k_{2 \theta}, \ldots, k_{r \theta}$ and $k_{1 \varphi}, k_{2 \varphi}, \ldots, k_{r \varphi}$ construct $\mathbf{K}_{\theta}$ and $\mathbf{K}_{\varphi}$, respectively. The $i$ th signal's polarization parameters are $k_{i \theta}$ and $k_{i \varphi}$, respectively. The signal vector is $\mathbf{S}(n)$. The covariance matrix of noise $\mathbf{W}(n)$ is

$$
E\left\{\mathbf{W}(n) \mathbf{W}(n)^{H}\right\}=\mathbf{Q}=\sigma^{2} \mathbf{I} .
$$

$(\cdot)^{H}$ denotes conjugate transpose of matrix $(\cdot) . \mathbf{I}$ is the identical matrix.

For simplicity, only the effect of mutual coupling among the same uniform linear array (ULA) is taken into consideration. The mutual coupling matrix (MCM) $\mathbf{C}$ is modeled as a banded symmetric Toeplitz matrix. $\mathbf{c}=\left[c_{1}, c_{2}, \ldots, c_{q}, 0, \ldots, 0\right]$ is the first row of $\mathbf{C}$. The element of vector $\mathbf{c}$ satisfying $0<$ $\left|c_{q}\right|<\left|c_{q-1}\right|<\cdots<c_{1}=1$. This matrix is formulated as

$$
\begin{aligned}
\mathbf{C} & =\text { Toeplitz }(\mathbf{c})=\text { Toeplitz }\left\{\left[c_{1}, c_{2}, \ldots, c_{q}, 0, \ldots, 0\right]\right\} \\
& =\left[\begin{array}{cccccc}
1 & c_{1} & \cdots & c_{q} & \cdots & 0 \\
c_{1} & 1 & c_{1} & \cdots & \ddots & 0 \\
\vdots & c_{1} & 1 & \ddots & \cdots & c_{q} \\
c_{q} & \cdots & \ddots & \ddots & c_{1} & \vdots \\
0 & \ddots & \cdots & c_{1} & 1 & c_{1} \\
0 & \cdots & c_{q} & \cdots & c_{1} & 1
\end{array}\right]
\end{aligned}
$$

Collecting $N$ snapshots, we have the matrix form of (5) as

$$
\mathbf{X}=\mathbf{C B S}+\mathbf{W} .
$$

There is a characteristic called "shadow effect" which most conformal arrays possess. It means that when the incident signal impinges on the array, not all elements could receive it. In order to solve this problem, the subarray divided technique (SDT) proposed in $[18,19]$ is adopted in this paper. The whole conformal array consists of several subarrays, and each subarray covers a certain range of angle. For the single curvature and symmetry of the cylinder, the parameter estimation mechanism and array design of each subarray are identical. Thus only one subarray is considered in this paper.

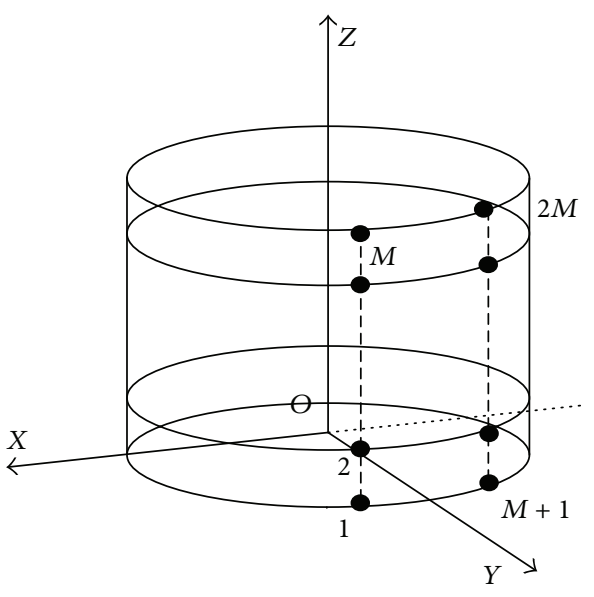

FIGURE 2: The structure of cylindrical conformal array.

\section{The Mechanism of Mutual Coupling Elimination}

The design of cylindrical conformal array is shown in Figure 2, the distance between two elements in the identical plane is $\lambda / 2$, and the distance between two neighbouring planes is $\lambda / 2$. The radius of the cylinder is $5 \lambda$.

According the form of the MCM $\mathbf{C}$, a mutual coupling elimination mechanism is introduced based on instrumental elements [26]. To show this mechanism in an intuitive way, we give an example based on $1 \sim M$ elements. The position vector $\mathbf{p}_{m}$ of the $m$ th element can be expressed as

$$
\mathbf{p}_{m}=x_{m} \overrightarrow{\mathbf{e}}_{x}+y_{m} \overrightarrow{\mathbf{e}}_{y}+z_{m} \overrightarrow{\mathbf{e}}_{z} .
$$

Since $M$ elements are arranged on the same generatrix, the $x$-coordinate has the relationship $x_{1}=x_{2}=\cdots=x_{M}=x$ as well as $y_{1}=y_{2}=\cdots=y_{M}=y$ and $h_{1}=h_{2}=$ $\cdots=h_{M}$. The manifold matrix of this subarray is $\mathbf{B}_{1}=$ $\left[\mathbf{b}_{1}\left(\theta_{1}\right), \mathbf{b}_{1}\left(\theta_{2}\right), \ldots, \mathbf{b}_{1}\left(\theta_{P}\right)\right]$, and the steering vector is

$$
\mathbf{b}_{1}\left(\theta_{p}\right)=\left[H_{p}, H_{p} v_{p}^{1}, \ldots, H_{p} v_{p}^{M-1}\right]^{T},
$$

where $v_{p}=\exp \left(-j 2 \pi d \cos \left(\theta_{p}\right) / \lambda\right)$ and

$$
H_{p}=h_{1} e^{-j 2 \pi\left(\left(x \sin \left(\theta_{p}\right) \cos \left(\varphi_{p}\right)+y \sin \left(\theta_{p}\right) \sin \left(\varphi_{p}\right)\right) / \lambda\right)} .
$$

The instrumental elements are set as the first and last $q$ elements. Although the array aperture is reduced, the effect of mutual coupling is eliminated as well. We construct a selection matrix that has the following form: $\mathbf{F}=$ $\left[\mathbf{0}_{(M-2 q) \times q} \mathbf{I}_{M-2 q} \mathbf{0}_{(M-2 q) \times q}\right]$, and the received data $\overline{\mathbf{X}}$ of center array can be given by

$$
\overline{\mathbf{X}}=\mathbf{P C B}_{1} \mathbf{S}+\mathbf{P W}=\overline{\mathbf{C}} \mathbf{B}_{1} \mathbf{S}+\mathbf{P W},
$$

where the new $\bar{M} \times M \overline{\mathbf{C}}$ is expressed as

$$
\overline{\mathbf{C}}=\mathbf{P C}=\left[\begin{array}{cccccccc}
c_{q} & \cdots & 1 & \cdots & c_{q} & 0 & \cdots & 0 \\
0 & c_{q} & \cdots & 1 & \cdots & c_{q} & \cdots & 0 \\
\vdots & \ddots & \ddots & \cdots & \ddots & \cdots & \ddots & \vdots \\
0 & \cdots & 0 & c_{q} & \cdots & 1 & \cdots & c_{q}
\end{array}\right]
$$


By using instrumental elements, we can get an important equation as follows:

$$
\begin{aligned}
\overline{\mathbf{C}}_{1}\left(\theta_{p}\right) & =\left[\begin{array}{c}
H_{p}\left(c_{q} v_{p}^{-q}+\cdots+c_{1} v_{p}^{-q}+1+c_{1} v_{p}+\cdots+c_{q} v_{p}^{q}\right) \\
H_{p}\left(c_{q} v_{p}^{1-q}+\cdots+c_{1}+v_{p}+c_{1} v_{p}^{2}+\cdots+c_{q} v_{p}^{q+1}\right) \\
\vdots \\
H_{p}\left(c_{q} v_{p}^{M-q-2}+\cdots+c_{1} v_{p}^{M-2}+v_{p}^{M-1}+c_{1} v_{p}^{M}+\cdots+c_{q} v_{p}^{M+q-2}\right) \\
H_{p}\left(c_{q} v_{p}^{M-q-1}+\cdots+c_{1} v_{p}^{M-1}+v_{p}^{M}+c_{1} v_{p}^{M+1}+\cdots+c_{q} v_{p}^{M+q-1}\right)
\end{array}\right] \\
= & H_{p}\left(c_{q} v_{p}^{-q}+\cdots+c_{1} v_{p}^{-1}+1+c_{1} v_{p}+\cdots+c_{q} v_{p}^{q}\right)\left[\begin{array}{c}
1 \\
v_{p} \\
\vdots \\
v_{p}^{M-1}
\end{array}\right] \\
= & \left(\begin{array}{c}
2 H_{p} \sum_{q^{\prime}=1}^{q} c_{q^{\prime}} \cos \left(\frac{2 q^{\prime} \pi \sin \left(\theta_{p}\right) d}{\lambda}\right)+1 \\
\bar{b}_{1}\left(\theta_{p}\right)=c\left(\theta_{p}\right) \overline{\mathbf{b}}_{1}\left(\theta_{p}\right)
\end{array}\right],
\end{aligned}
$$

where $\overline{\mathbf{b}}_{1}\left(\theta_{p}\right)$ is the ideal steering vector of the center array $(q+1 \sim M-q$ elements $), \bar{M}=M-2 p$, and $c\left(\theta_{p}\right)$ is a scalar which can be written as

$$
c\left(\theta_{p}\right)=\left(2 \sum_{q^{\prime}=1}^{q} c_{q^{\prime}} \cos \left(\frac{2 q^{\prime} \pi \sin \left(\theta_{p}\right) d}{\lambda}\right)+1\right) .
$$

Based on the (21), the new covariance matrix can be expressed as

$$
\begin{aligned}
\overline{\mathbf{R}}_{x} & =E\left\{\overline{\mathbf{X X}}^{H}\right\}=\overline{\mathbf{C}} \mathbf{B}_{1} \overline{\mathbf{R}}_{s} \mathbf{B}_{1}^{H} \overline{\mathbf{C}}^{H}+\sigma^{2} \mathbf{I} \\
& =\overline{\mathbf{B}}_{1} \mathbf{D} \overline{\mathbf{R}}_{s} \mathbf{D}^{H} \overline{\mathbf{B}}_{1}^{H}+\sigma^{2} \mathbf{I},
\end{aligned}
$$

where

$$
\begin{gathered}
\overline{\mathbf{B}}_{1}=\left[\overline{\mathbf{b}}_{1}\left(\theta_{1}\right), \overline{\mathbf{b}}_{1}\left(\theta_{2}\right), \ldots, \overline{\mathbf{b}}_{1}\left(\theta_{P}\right)\right], \\
\mathbf{D}=\operatorname{diag}\left\{c\left(\theta_{1}\right), c\left(\theta_{2}\right), \ldots, c\left(\theta_{P}\right)\right\} .
\end{gathered}
$$

Then (24) can be written as

$$
\overline{\mathbf{R}}_{x}=\overline{\mathbf{B}}_{1} \mathbf{R} \overline{\mathbf{B}}_{1}^{H}+\sigma^{2} \mathbf{I},
$$

where

$$
\mathbf{R}=\operatorname{diag}\left\{\sigma_{1}^{2}\left|c\left(\theta_{1}\right)\right|^{2}, \sigma_{2}^{2}\left|c\left(\theta_{2}\right)\right|^{2}, \ldots, \sigma_{P}^{2}\left|c\left(\theta_{P}\right)\right|^{2}\right\}
$$

and $\sigma_{p}^{2}$ stands for the power of $p$ th incident signal. A very interesting result is shown in (27) where the mutual coupling coefficients are coming into the novel signal covariance matrix completely. When $c\left(\theta_{p}\right) \neq 0, p=1,2, \ldots, P$, $\operatorname{rank}(\mathbf{R})=P$, the elimination of effect of mutual coupling can be accomplished. When $c\left(\theta_{p}\right)=0, p=1,2, \ldots, P$, we have

$$
c\left(\theta_{p}\right) \overline{\mathbf{b}}\left(\theta_{p}\right)=0
$$

Equation (33) implies that the center array is blind at some particular angles. It could not receive incident signal from certain directions. Thus, these angles are called "blind angles." Fortunately, for given mutual coefficients, $c\left(\bar{\theta}_{l}\right)$ is a continuous function, the probability of $c\left(\bar{\theta}_{l}\right)=0$ is approximate to zero, which means that "blind angles" phenomenon rarely happens in practice. More details can be found in [27].

As shown in Figure 3, there are two rotational invariance relations in the designed array. The ESPRIT algorithm which is similar to the algorithm described in [17] can be used for DOA estimation. The parameter pairing between elevation and azimuth has to be considered. However, the algorithm is not suitable for estimating conformal array. Based on PARAFAC theory, a novel high-accuracy 2D-DOA estimation algorithm is proposed in this paper for conformal array.

\section{DOA Estimation Based on PARAFAC}

When the elimination of mutual coupling is done, the decoupling between polarization and direction can be completed 


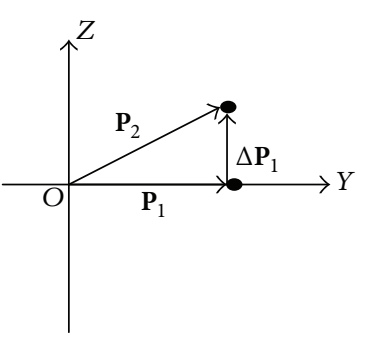

(a)

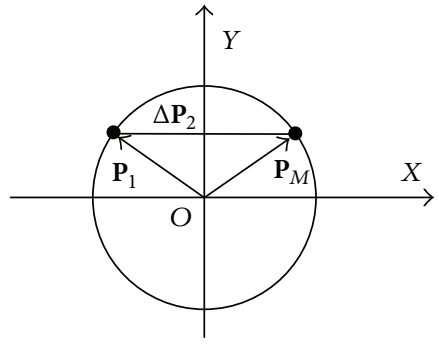

(b)

Figure 3: The distance vector. (a) The distance vector $\Delta \mathbf{P}_{1}$ between array 1 and array 2. (b) The distance vector $\Delta \mathbf{P}_{2}$ between array 3 and array 4 .

based on well array design. Define two $(\bar{M}-1) \times \bar{M}$ selection matrices:

$$
\begin{aligned}
& \mathbf{P}_{1}=\left[\begin{array}{cccc}
1 & \cdots & 0 & 0 \\
\vdots & \ddots & \vdots & \vdots \\
0 & \cdots & 1 & 0
\end{array}\right], \\
& \mathbf{P}_{2}=\left[\begin{array}{cccc}
0 & 1 & \cdots & 0 \\
\vdots & \vdots & \ddots & \vdots \\
0 & 0 & \cdots & 1
\end{array}\right] .
\end{aligned}
$$

$q+1 \sim M-q-1, q+2 \sim M-q, q+1 \sim M-q$, and $M+q+1 \sim 2 M-q$ elements constitute array 1, array 2, array 3, and array 4 , respectively. The distance vector between arrays 1 and 2 is $\Delta \mathbf{P}_{1}$ and $d_{1}=\left|\Delta \mathbf{P}_{1}\right|=\lambda / 2$. The distance vector between arrays 1 and 3 is $\Delta \mathbf{P}_{2}$ and $d_{2}=\left|\Delta \mathbf{P}_{2}\right|=\lambda / 2$, which is shown in Figure 3. Since the direction pattern is identical through one generatrix, the effect of polarization parameter can be ignored.

The distance among different subarrays is shown in Figures 2 and 3. In the global coordinate, the elevation and azimuth of $\Delta \mathbf{P}_{1}$ and $\Delta \mathbf{P}_{2}$ can be represented as $\theta_{\Delta \mathbf{P}_{1}}=0$, $\varphi_{\Delta \mathbf{P}_{1}}=\pi / 2$, and $\theta_{\Delta \mathbf{P}_{2}}=\pi / 2, \varphi_{\Delta \mathbf{P}_{2}}=0$, respectively.

Assume that $\mathbf{X}_{1}, \mathbf{X}_{2}, \mathbf{X}_{3}, \mathbf{X}_{4}$ represent the constructed data of array 1, array 2, array 3 , and array 4 , respectively. The origin of the coordinate is the reference point. So $\mathbf{X}_{1}, \mathbf{X}_{2}, \mathbf{X}_{3}, \mathbf{X}_{4}$ can be expressed as

$$
\begin{aligned}
& \mathbf{X}_{1}=\mathbf{B S}+\mathbf{N}_{1}, \\
& \mathbf{X}_{2}=\mathbf{B} \Psi_{1} \mathbf{S}+\mathbf{N}_{2}, \\
& \mathbf{X}_{3}=\mathbf{B} \Psi_{2} \mathbf{S}+\mathbf{N}_{3}, \\
& \mathbf{X}_{4}=\mathbf{B} \Psi_{1} \Psi_{2} \mathbf{S}+\mathbf{N}_{4} .
\end{aligned}
$$

The covariance matrices among the received data are

$$
\begin{aligned}
& \mathbf{R}_{1}=E\left\{\mathbf{X}_{1} \mathbf{X}_{1}^{H}\right\}=\mathbf{B} \mathbf{R}_{s} \mathbf{B}^{H}+\mathbf{Q}_{1}, \\
& \mathbf{R}_{2}=E\left\{\mathbf{X}_{2} \mathbf{X}_{1}^{H}\right\}=\mathbf{B} \Psi_{1} \mathbf{R}_{s} \mathbf{B}^{H}+\mathbf{Q}_{2}, \\
& \mathbf{R}_{3}=E\left\{\mathbf{X}_{3} \mathbf{X}_{1}^{H}\right\}=\mathbf{B} \Psi_{2} \mathbf{R}_{s} \mathbf{B}^{H}+\mathbf{Q}_{3}, \\
& \mathbf{R}_{4}=E\left\{\mathbf{X}_{4} \mathbf{X}_{1}^{H}\right\}=\mathbf{B} \Psi_{1} \Psi_{2} \mathbf{R}_{s} \mathbf{B}^{H}+\mathbf{Q}_{4},
\end{aligned}
$$

where $\mathbf{B}=\mathbf{P}_{1} \overline{\mathbf{B}}_{1}, \mathbf{R}_{s}=\operatorname{diag}\left\{s_{1}^{2}, \ldots, s_{r}^{2}\right\}$ represents the signal covariance matrix, and the noise covariance matrices are $\mathbf{Q}_{1} \sim \mathbf{Q}_{4}$, respectively:

$$
\begin{aligned}
& \boldsymbol{\Phi}_{1}=\operatorname{diag}\left[\exp \left(-j \omega_{11}\right), \exp \left(-j \omega_{12}\right), \ldots, \exp \left(-j \omega_{1 r}\right)\right], \\
& \boldsymbol{\Phi}_{2}=\operatorname{diag}\left[\frac{h_{3}\left(\theta_{1}, \varphi_{1}\right)}{h_{1}\left(\theta_{1}, \varphi_{1}\right)} \exp \left(-j \omega_{21}\right), \frac{h_{3}\left(\theta_{2}, \varphi_{2}\right)}{h_{1}\left(\theta_{2}, \varphi_{2}\right)}\right. \\
& \left.\cdot \exp \left(-j \omega_{22}\right), \ldots, \frac{h_{3}\left(\theta_{r}, \varphi_{r}\right)}{h_{1}\left(\theta_{r}, \varphi_{r}\right)} \exp \left(-j \omega_{2 r}\right)\right], \\
& \omega_{1 i}=\left(\frac{2 \pi}{\lambda}\right) \Delta \mathbf{P}_{1} \cdot \mathbf{u}_{i}=\left(\frac{2 \pi d_{1}}{\lambda}\right)\left[\sin \left(\theta_{\Delta \mathbf{P}_{1}}\right) \cos \left(\varphi_{\Delta \mathbf{P}_{1}}\right)\right. \\
& \cdot \sin \left(\theta_{i}\right) \cos \left(\varphi_{i}\right)+\sin \left(\theta_{\Delta \mathbf{P}_{1}}\right) \sin \left(\varphi_{\Delta \mathbf{P}_{1}}\right) \sin \left(\theta_{i}\right) \\
& \left.\quad \cdot \sin \left(\varphi_{i}\right)+\cos \left(\theta_{\Delta \mathbf{P}_{1}}\right) \cos \left(\theta_{i}\right)\right], \\
& \omega_{2 i}=\left(\frac{2 \pi}{\lambda}\right) \Delta \mathbf{P}_{2} \cdot \mathbf{u}_{i}=\left(\frac{2 \pi d_{2}}{\lambda}\right)\left[\sin \left(\theta_{\Delta \mathbf{P}_{2}}\right) \cos \left(\varphi_{\Delta \mathbf{P}_{2}}\right)\right. \\
& \quad \cdot \sin \left(\theta_{i}\right) \cos \left(\varphi_{i}\right)+\sin \left(\theta_{\Delta \mathbf{P}_{2}}\right) \sin \left(\varphi_{\Delta \mathbf{P}_{2}}\right) \sin \left(\theta_{i}\right) \\
& \left.\quad \cdot \sin \left(\varphi_{i}\right)+\cos \left(\theta_{\Delta \mathbf{P}_{2}}\right) \cos \left(\theta_{i}\right)\right],
\end{aligned}
$$

where $\theta_{\Delta \mathbf{P}_{i}}$ and $\varphi_{\Delta \mathbf{P}_{i}}(i=1,2)$ represent the elevation and azimuth of the distance vector in the global coordinate, respectively.

On the basis of the PARAFAC theory [11], the $m \times$ $m \times 4$ three-way array of the cylindrical conformal array is constructed by (34):

$$
\left|\begin{array}{l}
\mathbf{R}(:,:, 1) \\
\mathbf{R}(:,:, 2) \\
\mathbf{R}(:,:, 3) \\
\mathbf{R}(:,:, 4)
\end{array}\right|=\left|\begin{array}{l}
\mathbf{R}_{1} \\
\mathbf{R}_{2} \\
\mathbf{R}_{3} \\
\mathbf{R}_{4}
\end{array}\right|=\left|\begin{array}{c}
\mathbf{B R}_{s} \mathbf{B}^{H} \\
\mathbf{B} \Psi_{1} \mathbf{R}_{s} \mathbf{B}^{H} \\
\mathbf{B} \Psi_{2} \mathbf{R}_{s} \mathbf{B}^{H} \\
\mathbf{B} \Psi_{1} \Psi_{2} \mathbf{R}_{s} \mathbf{B}^{H}
\end{array}\right|+\widetilde{\mathbf{Q}},
$$

where $\mathbf{R}_{i}(i=1, \ldots, 4)$ are the sample covariance matrices, respectively, and $\widetilde{\mathbf{Q}}$ represents the noise in real observation. 
Let $\mathbf{C}=\mathbf{B}^{H}$, and, based on the definition of Khatri-Rao product, (36) can be transformed as

$$
\begin{aligned}
\mathbf{R} & =(\mathbf{D} \odot \mathbf{B}) \mathbf{C}+\widetilde{\mathbf{Q}}, \\
\mathbf{R}_{X} & =\left(\mathbf{C}^{T} \odot \mathbf{D}\right) \mathbf{B}^{T}+\widetilde{\mathbf{Q}}_{X}, \\
\mathbf{R}_{Y} & =\left(\mathbf{B} \odot \mathbf{C}^{T}\right) \mathbf{D}^{T}+\widetilde{\mathbf{Q}}_{Y}, \\
\mathbf{D} & =\left[\begin{array}{c}
\Lambda^{-1}\left(\mathbf{R}_{s}\right) \\
\Lambda^{-1}\left(\Psi_{1} \mathbf{R}_{s}\right) \\
\Lambda^{-1}\left(\Psi_{2} \mathbf{R}_{s}\right) \\
\Lambda^{-1}\left(\Psi_{1} \Psi_{2} \mathbf{R}_{s}\right)
\end{array}\right],
\end{aligned}
$$

where $\Lambda^{-1}\left(\mathbf{R}_{s}\right)$ is the row vector constructed by the diagonal entries of the diagonal matrix $\mathbf{R}_{s}$.

On the basis of noisy observation, problem (37) can be transformed into solving a least square problem:

$$
\min _{\mathbf{D}, \mathbf{B}, \mathbf{C}}\|\mathbf{R}-(\mathbf{D} \odot \mathbf{B}) \mathbf{C}\|_{F}^{2} .
$$

The principle of alternating least squares (ALS) can be used to fit the problem in (41). In the noiseless condition, ALS can be used to solve the matrices $\mathbf{B}, \mathbf{C}$, and $\mathbf{D}$ which constructed the three-way array $\mathbf{R}$. The least square estimation of matrix C can be expressed as

$$
\mathbf{C}=\arg \min _{\mathbf{C}}\|\mathbf{R}-(\mathbf{D} \odot \mathbf{B}) \mathbf{C}\|_{F}^{2} .
$$

Similarly, the matrices $\mathbf{B}$ and $\mathbf{D}$ can be expressed as

$$
\begin{aligned}
& \mathbf{B}^{T}=\arg \min _{\mathbf{B}}\left\|\mathbf{R}_{X}-\left(\mathbf{C}^{T} \odot \mathbf{D}\right) \mathbf{B}^{T}\right\|_{F}^{2}, \\
& \mathbf{D}^{T}=\arg \min _{\mathbf{D}}\left\|\mathbf{R}_{Y}-\left(\mathbf{B} \odot \mathbf{C}^{T}\right) \mathbf{D}^{T}\right\|_{F}^{2} .
\end{aligned}
$$

In the iterative procedure, given matrices $\mathbf{B}$ and $\mathbf{D}$, the matrix C can be represented as

$$
\mathbf{C}=(\mathbf{D} \odot \mathbf{B})^{\dagger} \mathbf{R} .
$$

The expression of matrices $\mathbf{B}^{T}$ and $\mathbf{D}^{T}$ is

$$
\begin{aligned}
& \mathbf{B}^{T}=\left(\mathbf{C}^{T} \odot \mathbf{D}\right)^{\dagger} \mathbf{R}_{X}, \\
& \mathbf{D}^{T}=\left(\mathbf{B} \odot \mathbf{C}^{T}\right)^{\dagger} \mathbf{R}_{Y},
\end{aligned}
$$

where $(\cdot)^{\dagger}$ denotes the pseudoinverse of matrix $(\cdot)$.

The matrix $\mathbf{D}$ can be acquired by the TALS algorithm. $\omega_{1 i}$ and $\omega_{2 i}$ can be calculated by matrix $\mathbf{D}$ :

$$
\omega_{1 i}=-\frac{1}{2}\left(\operatorname{angle}\left\lceil\frac{\mathbf{D}_{2 i}}{\mathbf{D}_{1 i}}\right\rceil+\text { angle }\left\lceil\frac{\mathbf{D}_{4 i}}{\mathbf{D}_{3 i}}\right\rceil\right)
$$

$[\cdot]$ stands for the matrix and $[\cdot]$ stands for the absolute value operation, where $\mathbf{D}_{j i}$ represents the $j$ th row of $\mathbf{D}$. Because $h_{1}$, $h_{3}$, and $h_{4}$ are real numbers, $\mathbf{D}_{3 i} / \mathbf{D}_{1 i}$ and $\mathbf{D}_{4 i} / \mathbf{D}_{2 i}$ are squared to solve the ambiguity caused by the positive and negative values of $h_{1}, h_{3}$, and $h_{4}$ :

$$
\begin{aligned}
\omega_{2 i} & =-\frac{1}{2} \text { angle }\left(\left[\frac{h_{3}\left(\theta_{i}, \varphi_{i}\right)}{h_{1}\left(\theta_{i}, \varphi_{i}\right)} \exp \left(-j \omega_{2 i}\right)\right]^{2}\right) \\
& =-\frac{1}{2} \text { angle }\left(\exp \left(-j 2 \omega_{2 i}\right)\right)=-\frac{1}{2} \operatorname{angle}\left(\left[\frac{\mathbf{D}_{3 i}}{\mathbf{D}_{1 i}}\right]^{2}\right) \\
& =-\frac{1}{2} \text { angle }\left(\left[\frac{\mathbf{D}_{4 i}}{\mathbf{D}_{2 i}}\right]^{2}\right),
\end{aligned}
$$

and then

$$
\omega_{2 i}=-\frac{1}{4}\left|\operatorname{angle}\left(\left[\frac{\mathbf{D}_{3 i}}{\mathbf{D}_{1 i}}\right]^{2}\right)+\operatorname{angle}\left(\left[\frac{\mathbf{D}_{4 i}}{\mathbf{D}_{2 i}}\right]^{2}\right)\right|
$$

The elevation $\theta_{i}$ and azimuth $\varphi_{i}$ of $i$ th incident signal can be expressed as

$$
\begin{aligned}
\theta_{i} & =\arccos \left(\frac{\lambda \omega_{1 i}}{2 \pi d_{2}}\right)=\arccos \left(\frac{2 \omega_{1 i}}{\pi}\right), \\
\varphi_{i} & =\arccos \left(\frac{\lambda \omega_{2 i}}{2 \pi d_{2} \sin \left(\theta_{i}\right)}\right) \\
& =\arccos \left(\frac{2 \omega_{2 i}}{\pi \sin \left(\theta_{i}\right)}\right) .
\end{aligned}
$$

\section{Mutual Coupling Coefficient Estimation}

Based on the 2D-DOA estimation of incident signals, the estimations of mutual coupling coefficients can be obtained. The covariance matrix of $\mathbf{X}$ can be expressed as

$$
\mathbf{R}_{X}=E\left\{\mathbf{X} \mathbf{X}^{H}\right\}=\mathbf{C A R}_{s} \mathbf{A}^{H} \mathbf{C}^{H}+\sigma_{n}^{2} \mathbf{I}_{N} .
$$

The rank of $\mathbf{C A R}_{s} \mathbf{A}^{H} \mathbf{C}^{H}$ is $P$. Take the eigendecomposition of $\mathbf{R}_{X}$; the $P$ big eigenvalues $\lambda_{1}, \lambda_{2}, \ldots, \lambda_{P}$ and $M-P$ small eigenvalues $\lambda_{P+1}=\lambda_{P+2}=\cdots=\lambda_{K}=\sigma_{n}^{2}$ can be obtained, respectively. Their corresponding eigenvectors are $\mathbf{u}_{1}, \mathbf{u}_{2}, \ldots, \mathbf{u}_{M}$. The signal subspace is spanned by $\mathbf{C A}$, which is orthogonal to the noise subspace spanned by $\mathbf{u}_{K_{u}+P+1}, \mathbf{u}_{K_{u}+P+2}, \ldots, \mathbf{u}_{M}$ :

$$
\mathbf{U}_{n}^{H} \mathbf{C a}\left(\theta_{i}\right)=0, \quad i=1,2, \ldots, P .
$$


By considering the complex symmetric Toeplitz form of $\mathbf{C}$, $\mathbf{C a}(\theta)$ can be expressed as

$$
\begin{gathered}
\mathbf{C a}(\theta)=\left[\begin{array}{cccc}
1 & c_{1} & \cdots & c_{N-1} \\
c_{1} & 1 & \cdots & c_{N-2} \\
\vdots & \vdots & \vdots & \vdots \\
c_{N-1} & c_{N-2} & \cdots & 1
\end{array}\right]\left[\begin{array}{c}
1 \\
v \\
\vdots \\
v^{M-1}
\end{array}\right] \\
=\left[\begin{array}{ccccc}
1 & v & \cdots & v^{M-2} & v^{M-1} \\
v & 1+v^{2} & \cdots & v^{M-1} & 0 \\
v^{2} & v+v^{3} & \cdots & 0 & 0 \\
\vdots & \vdots & \ddots & \vdots & 0 \\
v^{M-2} & v^{M-3}+v^{M-1} & \cdots & 1 & 0 \\
v^{M-1} & v^{M-2} & \cdots & v & 1
\end{array}\right]\left[\begin{array}{c}
1 \\
c_{1} \\
\vdots \\
c_{N-1}
\end{array}\right] \\
=\mathbf{T}(\theta) \mathbf{c}, \quad
\end{gathered}
$$

where $\mathbf{T}(\theta)$ is the sum of two $M \times\left(M_{0}+1\right)$ matrices [28].

Based on (52) and the estimations of $\theta_{1}, \theta_{2}, \ldots, \theta_{P}$, we have

$$
\mathbf{U}_{n}^{H} \mathbf{T}\left(\theta_{i}\right) \mathbf{c}=0, \quad i=1,2, \ldots, P
$$

It can be seen that (54) is the linear equations of mutual coupling coefficients $\mathbf{c}$. The coefficient matrix can be defined as

$$
\mathbf{Q}=\left[\begin{array}{c}
\mathbf{U}_{n}^{H} \mathbf{T}\left(\theta_{1}\right) \\
\vdots \\
\mathbf{U}_{n}^{H} \mathbf{T}\left(\theta_{P}\right)
\end{array}\right] .
$$

Then (50) can be written as

$$
\mathbf{Q c}=0,
$$

where $\mathbf{Q}$ is a $P(M-P) \times\left(M_{0}+1\right)$ matrix and $\mathbf{Q}=$ $\left[\mathbf{q}_{1}, \mathbf{q}_{2}, \ldots, \mathbf{q}_{P}\right]$. Due to $\mathbf{c}(1)=1$, we have

$$
\mathbf{Q} \mathbf{c}=\left[\mathbf{q}_{1}, \mathbf{q}_{2}, \ldots, \mathbf{q}_{M_{0}+1}\right]\left[\begin{array}{c}
1 \\
c_{1} \\
\vdots \\
c_{M_{0}}
\end{array}\right]=0 .
$$

When $P(M-P) \geq M_{0}-1$, the least square solution is obtained as

$$
\left[c_{1}, c_{2}, \ldots, c_{M_{0}}\right]=-\left[\mathbf{q}_{2}, \ldots, \mathbf{q}_{M_{0}+1}\right]^{\dagger} \mathbf{q}_{1} .
$$

The mutual coupling coefficients estimation is completed.

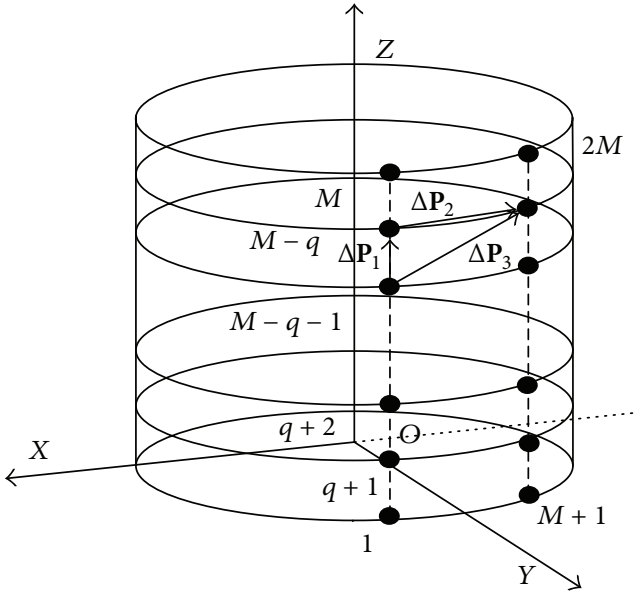

FIgURE 4: The distance vector between distinct subarrays.

\section{Discussion}

The estimators of $\mathbf{B}, \mathbf{C}$, and $\mathbf{D}$ have the same column permutation matrix; that is, the ith column of the steering matrix B corresponds to the $i$ th of the matrix $\mathbf{D}$. Thus, the elevation and azimuth pair with each other automatically.

When $P \geq 2$, the parameter pairing between $\omega_{1 i}(i=$ $1,2, \ldots, P)$ and $\omega_{2 i}(i=1,2, \ldots, P)$ is a big problem in [21]. Here, we give another method to solve the parameter pairing problem in [21]. Theoretically, the eigenvector of $\boldsymbol{\Phi}_{1}, \Phi_{2}$, and $\Phi_{3}$ is identical. However, the EVD of them are done independently. The order of eigenvector may be distinct. In order to obtain accurate DOA estimation, this problem must be solved. We construct another subarray: array 1 and array 5 constructed by $M+q+2 \sim 2 M-q$ elements. The distance vector is $\Delta \mathbf{P}_{3}$. Based on the relationship between $\Delta \mathbf{P}_{1}, \Delta \mathbf{P}_{2}$, and $\omega_{1 i}, \omega_{2 i}$, the relationship between $\Delta \mathbf{P}_{3}$ and $\omega_{3 k}(k=$ $1,2, \ldots, P)$ can be obtained similarly. As shown in Figure 4, $\Delta \mathbf{P}_{3}=\Delta \mathbf{P}_{1}+\Delta \mathbf{P}_{2}$.

For the identical source, we have

$$
\begin{aligned}
\text { angle } & \left(\left[\frac{h_{2}\left(\theta_{p}, \varphi_{p}\right)}{h_{1}\left(\theta_{p}, \varphi_{p}\right)} \exp \left(-j \omega_{3 p}\right)\right]^{2}\right) \\
= & \operatorname{angle}\left(\left(t_{3 k}\right)^{2}\right) \\
= & \operatorname{angle}\left(\exp \left(-j 2 \omega_{1 i}\right)\right)+\operatorname{angle}\left(\exp \left(-j 2 \omega_{2 i}\right)\right) \\
= & \operatorname{angle}\left(\left(t_{1 i}\right)^{2}\right)+\operatorname{angle}\left(\left(t_{2 j}\right)^{2}\right),
\end{aligned}
$$

where $t_{3 k}$ is the eigenvalue of $\Phi_{3} . \Phi_{3}$ can be solved easily according to the solution procedure of $\Phi_{1}$ and $\Phi_{2}$. Thus, the parameter pairing problem can be transformed as a problem which minimizes

$$
\begin{aligned}
\min & \mid \operatorname{angle}\left(\left(t_{3 k}\right)^{2}\right) \\
& -\left[\operatorname{angle}\left(\left(t_{1 i}\right)^{2}\right)+\operatorname{angle}\left(\left(t_{2 j}\right)^{2}\right)\right] \mid .
\end{aligned}
$$

Based on (60), the parameter pairing is completed finally. 


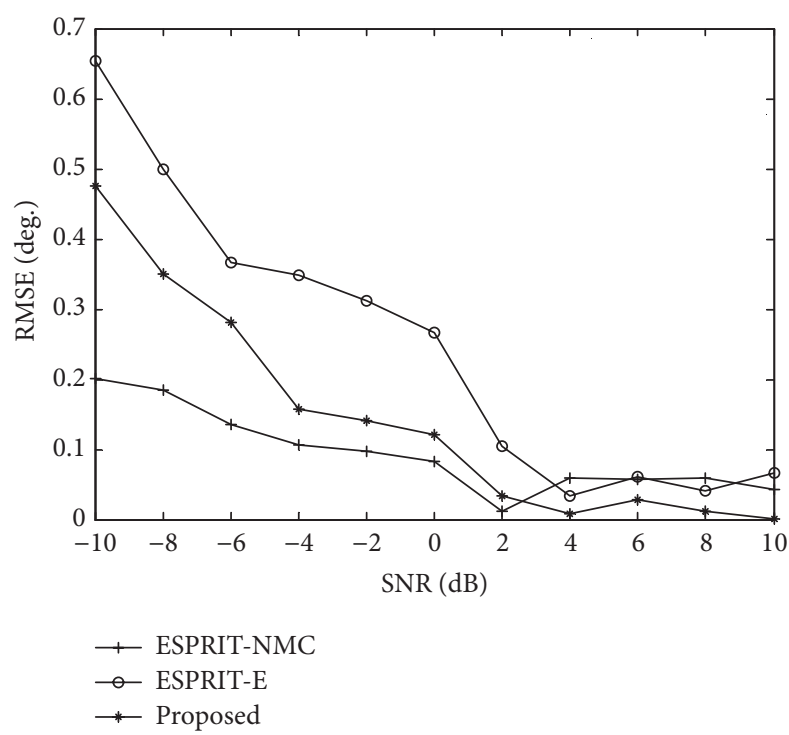

(a)

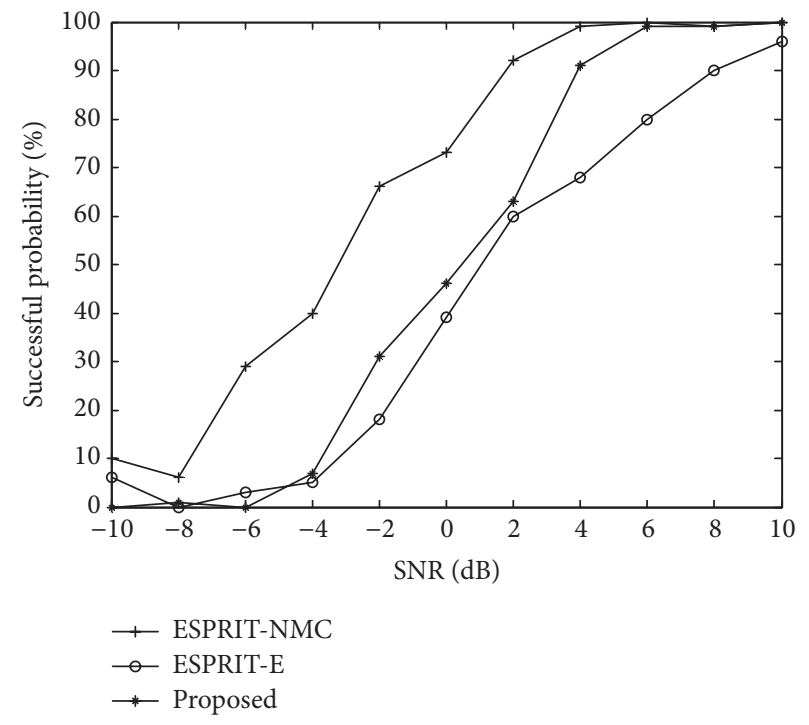

(b)

FIGURE 5: The performance against SNR (a). The RMSE against SNR (b). The successful probability against SNR.

The steps of DOA estimation for cylindrical conformal array in the presence of mutual coupling can be summarized as follows:

(1) Use the selection matrix $\mathbf{F}=$ $\left[\mathbf{0}_{(M-2 q) \times q} \mathbf{I}_{M-2 q} \mathbf{0}_{(M-2 q) \times q}\right]$ to eliminate the effect of mutual coupling.

(2) Set array 1 array 4 to construct three subarrays.

(3) Construct the PARAFAC model based on (36).

(4) Update (44), (45), and the matrix D can be obtained by TALS algorithm.

(5) Calculate $\omega_{1 i}$ and $\omega_{2 i}$ based on (46) and (47).

(6) Estimate the 2D-DOA for cylindrical conformal array based on (49) and (50).

(7) Calculate the covariance matrix of the data received by element $1 \sim M$ based on (51).

(8) Obtain the mutual coupling coefficients based on (58).

\section{Simulation Results}

In this section, we present numerical simulation results to illustrate the performance of the proposed algorithm. We compare the proposed algorithm with ESPRIT without mutual coupling (ESPRIT-NMC) [5] and the ESPRIT-E algorithm proposed in [21]. In this paper, root mean square error (RMSE) is calculated by the formula

$$
\begin{aligned}
\text { RMSE } & =\sqrt{\frac{1}{200} \sum_{\eta=1}^{200}\left[\left(\widehat{\theta}_{k, \eta}-\theta\right)^{2}+\left(\hat{\varphi}_{k, \eta}-\varphi\right)^{2}\right]}, \\
\operatorname{RMSE}_{c} & =\sqrt{\frac{1}{200\|c\|} \sum_{\eta=1}^{200}\|\widehat{c}(\eta)-c\| \times 100 \%,}
\end{aligned}
$$

where $\widehat{\theta}_{k, \eta}$ and $\widehat{\varphi}_{k, \eta}$ are the estimate of the elevation and azimuth of the $k$ th incident signal in the $\eta$ th trial. For the sake of simplicity, only the cylindrical conformal array is utilized for simulation, and the array structure is shown in Figure 2. The number of elements is 16 ; that is, $m=8$. Without loss of generality, $k_{1 \theta}=0.5, k_{1 \varphi}=0.5 ; k_{2 \theta}=0.3$, $k_{2 \varphi}=0.7$. The pattern transformation can be found in [25]. The azimuth and elevation of two incident signals are $\left(100^{\circ}\right.$, $\left.60^{\circ}\right)$ and $\left(95^{\circ}, 50^{\circ}\right)$, respectively. The freedom of degree (FOD) of mutual coupling is 2 , and $c_{1}=0.4500+i 0.5362$ and $c_{2}=$ $0.2598-i 0.1500$. Since the instrumental elements are used, the elements actually used are 12; that is, $m=6$. For ESPRITNMC, the elements actually used are 12 ; that is, $m=6.200$ Monte Carlo trials are considered in the simulations.

The RMSE and successful probability of different algorithms against SNR are shown in Figures 5(a) and 5(b), respectively. The snapshot number is 200 . It can be seen from Figure 5(a) that the RMSE of the proposed algorithm is smaller than that of ESPRIT-E. Since the mutual coupling coefficient has been estimated, this result can be used to recover the original covariance matrix. The array aperture is extended compared with ESPRIT-E and ESPRIT-NMC. It can be seen from Figure 5(b) that the successful probability increase as SNR increases. The successful probability of ESPRIT-E is higher than that of ESPRIT-NMC. The proposed algorithm outperforms ESPRIT-E and ESPRIT-NMC.

The RMSE and successful probability of different algorithms against snapshot number are shown in Figures 5(a) and 5(b), respectively. SNR is fixed at $0 \mathrm{~dB}$. It can be seen from Figure 6(a) that the RMSE of proposed algorithm is smaller than that of ESPRIT-E. When snapshot number is larger than 700, the RMSE of proposed algorithm approximates ESPRITNMC. The successful probability of proposed algorithm is higher than that of ESPRIT-E. However, the proposed algorithm does not reach $100 \%$ because of the low SNR $(0 \mathrm{~dB})$. 


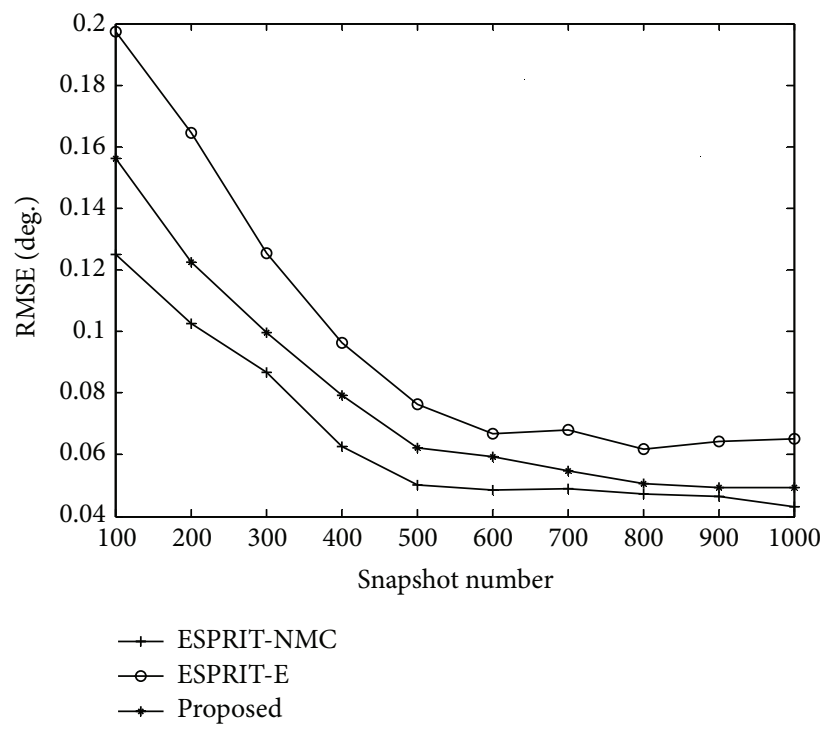

(a)

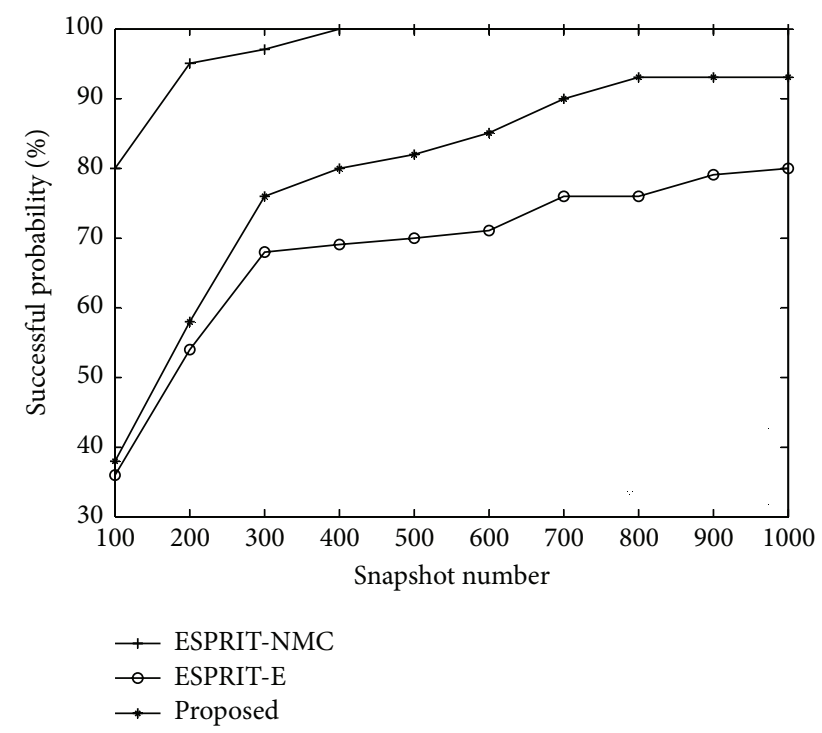

(b)

FIGURE 6: The performance against snapshot number (a). The RMSE against snapshot number (b). The successful probability against snapshot number.

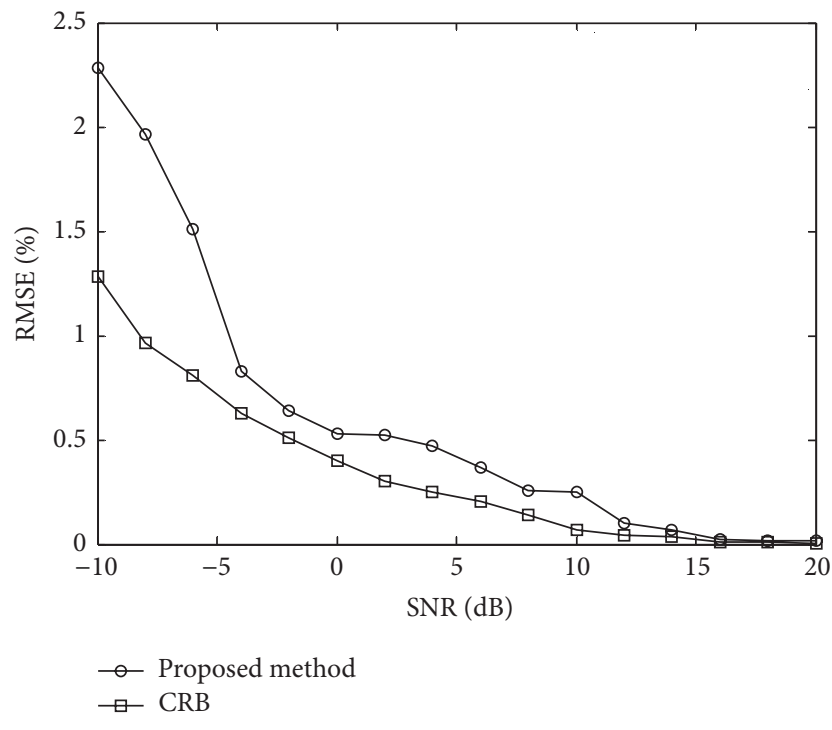

FIgURE 7: The RMSE against SNR.

Thus, the proposed algorithm has better performance compared with ESPRIT-E.

The mutual coupling estimation versus input SNR is shown in Figure 7. The results show that the mutual coupling has little influence on the proposed algorithm. Although some array apertures are used to eliminate the effect of mutual coupling, the proposed algorithm still has high estimation accuracy and approaches CRB at high SNR.

\section{Conclusions}

In this paper, a novel DOA and mutual coupling coefficient estimation algorithm for conformal array is proposed in
ITS. By constructing the spectical mutual coupling matrix (MCM), the effect of mutual coupling can be eliminated via instrumental element method. Then the DOA of incident signals can be estimated based on parallel factor (PARAFAC) theory. The proposed algorithm can also be extended to other conformal arrays. However, only the mutual coupling in the identical linear array is considered in this paper. The mutual coupling between different generatrices should be studied. The Cramér-Rao bound is also an interesting problem to research, and we will focus on it in the future work.

\section{Conflict of Interests}

The authors declare that there is no conflict of interests regarding the publication of this paper.

\section{Acknowledgments}

This work was supported in part by Qing Lan Project, by the National Science Foundation of China under Grant nos. 61572172, 61401147, 61301095, and 61201237, and by the Natural Science Foundation of JiangSu Province of China, no. BK20140248. This work was supported in part by the Key Development Program of Basic Research of China (JCKY2013604B001), Nature Science Foundation of Heilongjiang Province of China (nos. QC2012C069 and F201408), and the Fundamental Research Funds for the Central Universities (no. HEUCF1508).

\section{References}

[1] H. Krim and M. Viberg, "Two decades of array signal processing research: the parametric approach," IEEE Signal Processing Magazine, vol. 13, no. 4, pp. 67-94, 1996. 
[2] D. H. Johnson and D. E. Dudgeon, Array Signal ProcessingConcepts and Techniques, Prentice Hall, Englewood Cliffs, NJ, USA, 1993.

[3] P. Stocia and R. Moses, Introduction to Spectral Analysis, Prentice Hall, Upper Saddle River, NJ, USA, 2004.

[4] R. O. Schmidt, "Multiple emitter location and signal parameter estimation," IEEE Transactions on Antennas and Propagation, vol. 34, no. 3, pp. 276-280, 1986.

[5] R. Roy and T. Kailath, "ESPRIT-estimation of signal parameters via rotational invariance techniques," IEEE Transactions on Acoustics, Speech and Signal Processing, vol. 37, no. 7, pp. 984995, 1989.

[6] T. T. Zhang, Y. L. Lu, and H. T. Hui, "Compensation for the mutual coupling effect in uniform circular arrays for 2D DOA estimations employing the maximum likelihood technique," IEEE Transactions on Aerospace and Electronic Systems, vol. 44, no. 3, pp. 1215-1221, 2008.

[7] L. Wan, G. Han, J. J. P. C. Rodrigues, W. Si, and N. Feng, "An energy efficient DOA estimation algorithm for uncorrelated and coherent signals in virtual MIMO systems," Telecommunication Systems, vol. 59, no. 1, pp. 93-110, 2015.

[8] Z. T. Huang, Z. M. Liu, J. Liu, and Y. Y. Zhou, "Performance analysis of MUSIC for non-circular signals in the presence of mutual coupling," IET Radar, Sonar \& Navigation, vol. 4, no. 5, pp. 703-711, 2010.

[9] L. Wan, G. Han, L. Shu, and N. Feng, "The critical patients localization algorithm using sparse representation for mixed signals in emergency healthcare system," IEEE Systems Journal, 2015.

[10] N. D. Sidiropoulos, R. Bro, and G. B. Giannakis, "Blind PARAFAC receivers for DS-CDMA systems," IEEE Transactions on Signal Processing, vol. 48, no. 3, pp. 810-823, 2000.

[11] N. D. Sidiropoulos, R. Bro, and G. B. Giannakis, "Parallel factor analysis in sensor array processing," IEEE Transactions on Signal Processing, vol. 48, no. 8, pp. 2377-2388, 2000.

[12] J. L. Liang, "Joint azimuth and elevation direction finding using cumulant," IEEE Sensors Journal, vol. 9, no. 4, pp. 390-398, 2009.

[13] X.-F. Gong, Z.-W. Liu, and Y.-G. Xu, "Regularised parallel factor analysis for the estimation of direction-of-arrival and polarisation with a single electromagnetic vector-sensor," IET Signal Processing, vol. 5, no. 4, pp. 390-396, 2011.

[14] L. Josefsson and P. Persson, Conformal Array Antenna Theory and Design, IEEE Press, Piscataway, NJ, USA, 2006.

[15] W. T. Li, X. W. Shi, Y. Q. Hei, S. F. Liu, and J. Zhu, "A hybrid optimization algorithm and its application for conformal array pattern synthesis," IEEE Transactions on Antennas and Propagation, vol. 58, no. 10, pp. 3401-3406, 2010.

[16] W.-J. Si, L.-T. Wan, and Z.-X. Tian, "Fast DOA estimation based on cylindrical conformal array antenna," Systems Engineering and Electronics, vol. 35, no. 8, pp. 1589-1595, 2013.

[17] L. Wan, L. Liu, G. Han, and J. Rodrigues, "A low energy consumption DOA estimation approach for conformal array in ultra-wideband," Future Internet, vol. 5, no. 4, pp. 611-630, 2013.

[18] L. Wan, G. Han, J. Jiang, and L. Shu, "Distributed DOA estimation based on manifold separation technique in mobile wireless sensor networks," in Proceedings of the 2nd Workshop on Mobile Sensing, Computing and Communication (MSCC '15), pp. 1-6, ACM, Hangzhou, China, June 2015.

[19] L. Wan, G. Han, L. Shu, N. Feng, C. Zhu, and J. Lloret, "Distributed parameter estimation for mobile wireless sensor network based on cloud computing in battlefield surveillance system," IEEE Access, vol. 3, pp. 1729-1739, 2015.
[20] W. Si, L. Wan, L. Liu, Z. Tian, and X. Lan, "Real-time ultrawideband direction finding for the conformal array antenna," Journal of Harbin Engineering University, vol. 35, no. 7, pp. 913918, 2014.

[21] H. Feng, L. Liu, and B. Wen, "2D-DOA estimation for cylindrical array with mutual coupling," Mathematical Problems in Engineering, vol. 2014, Article ID 716978, 8 pages, 2014.

[22] W. Si, L. Wan, L. Liu, Z. Tian, and L. Li, "Direction-ofarrival estimation for arbitrary array configurations in ultrawideband," in Proceedings of the 2nd International Conference on Instrumentation, Measurement, Computer, Communication and Control (IMCCC '12), pp. 234-237, IEEE, Harbin, China, December 2012.

[23] Y. Zou, H. Xie, L. Wan, and G. Han, "High accuracy frequency and 2D-DOAs estimation of conformal array based on PARAFAC," Journal of Internet Technology, vol. 16, no. 1, pp. 107119, 2015.

[24] L.-T. Wan, L.-T. Liu, W.-J. Si, and Z.-X. Tian, "Joint estimation of 2D-DOA and frequency based on space-time matrix and conformal array," The Scientific World Journal, vol. 2013, Article ID 463828, 10 pages, 2013.

[25] L. Wan, W. Si, L. Liu, Z. Tian, and N. Feng, "High accuracy 2D-DOA estimation for conformal array using PARAFAC," International Journal of Antennas and Propagation, vol. 2014, Article ID 394707, 14 pages, 2014.

[26] X. Xu, Z. Ye, and Y. Zhang, "DOA estimation for mixed signals in the presence of mutual coupling," IEEE Transactions on Signal Processing, vol. 57, no. 9, pp. 3523-3532, 2009.

[27] J. Dai, W. Xu, and D. Zhao, "Real-valued DOA estimation for uniform linear array with unknown mutual coupling," Signal Processing, vol. 92, no. 9, pp. 2056-2065, 2012.

[28] Z. Ye and C. Liu, "On the resiliency of MUSIC direction finding against antenna sensor coupling," IEEE Transactions on Antennas and Propagation, vol. 56, no. 2, pp. 371-380, 2008. 

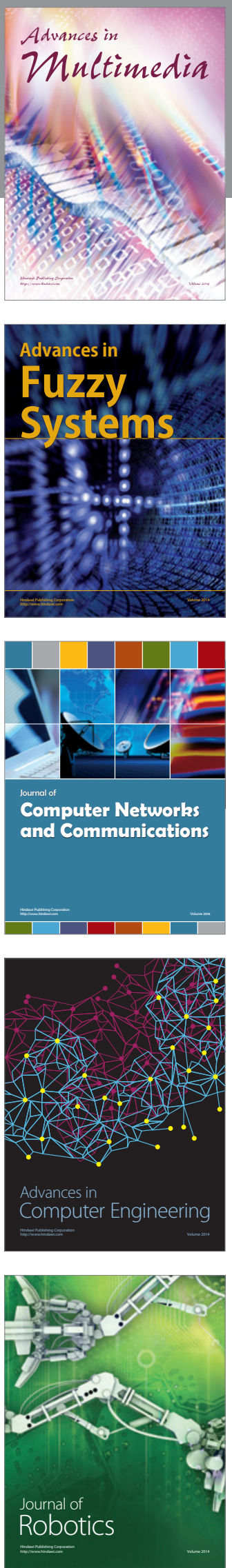

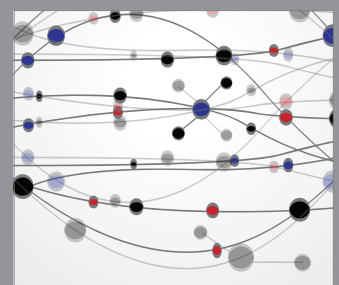

The Scientific World Journal
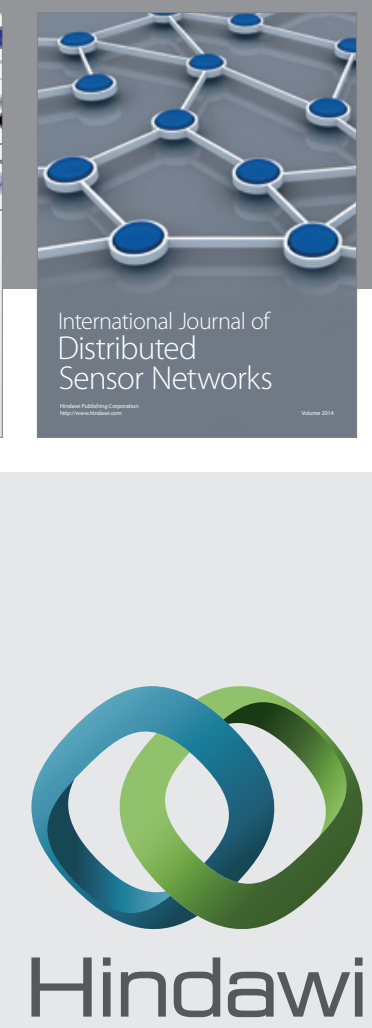

Submit your manuscripts at

http://www.hindawi.com
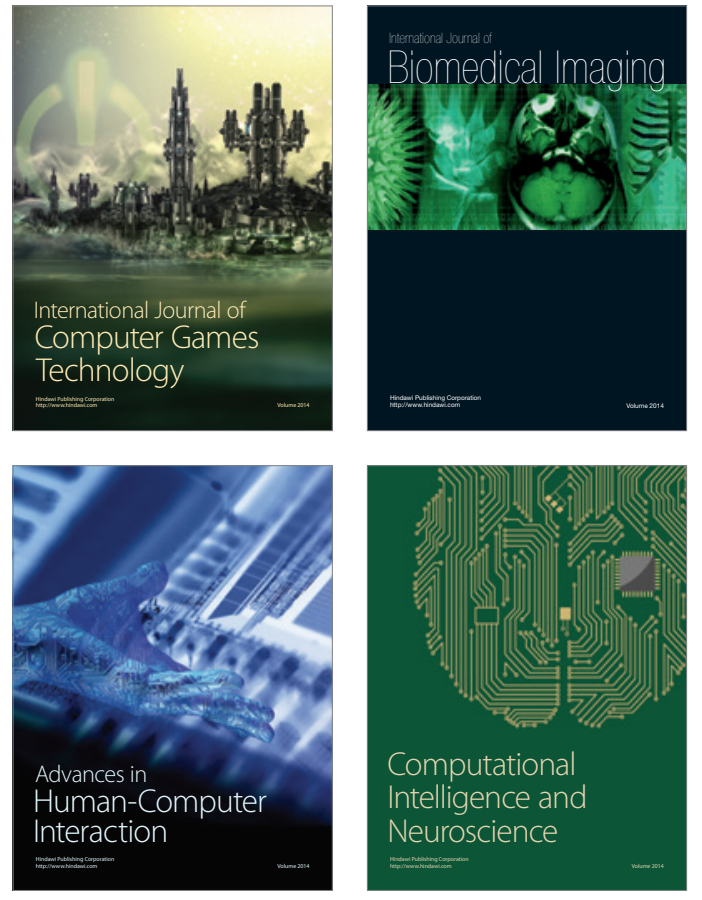
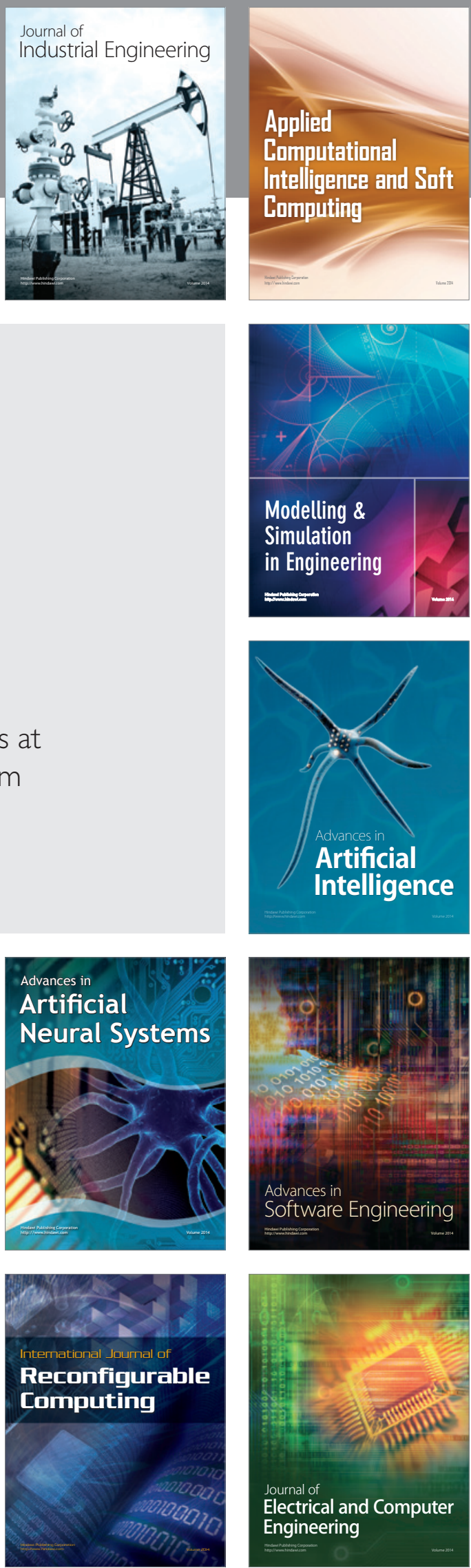